\title{
Linear response deviations in photopolymers
}

\author{
M. Ortuño ${ }^{1}$, C. Neipp ${ }^{1}$, E. Fernández ${ }^{2}$, M. L. Álvarez ${ }^{1}$, A. Beléndez ${ }^{1}$ \\ 1. Departamento de Física, Ingeniería de Sistemas y Teoría de la Señal \\ 2. Departamento de Óptica, Farmacología y Anatomía \\ Universidad de Alicante, 03690 San Vicente del Raspeig, Alicante, Spain \\ mos@ua.es
}

\begin{abstract}
In holographic recording photopolymers the diffraction efficiency develops according to Kogelnik expression during hologram recording stage. In the ideal situation, the curve of diffraction efficiency versus energetic exposure has a linear zone, although it is usual to find examples of deviations to this behavior. In this investigation we study different cases in which there are deviations to ideal behavior, proposing different hypothesis in order to explain the situations that can be experimentally obtained.
\end{abstract}

The more important deviation experimentally observed is when the first maximum is lower than the second one in the diffraction efficiency versus exposure curve during recording. A main hypothesis is presented, which is related to the dye concentration into the layer and the molecular weight of the polymer chains generated in the polymerization process.

Keywords: photopolymer, holographic recording materials, Kogelnik, volume holograms, diffraction gratings.

\section{Introduction}

Acrylamide (AA) photopolymers with a poly(vinyl alcohol) (PVA) binder are very versatile holographic recording materials [1]. Their most important characteristics include high diffraction efficiency, ease of preparation and the fact that they do not need post-processing after the hologram has been recorded [2]. These properties make them very suitable for studying factors relating to the process of hologram recording, or changes in the photopolymer composition. Recently, acrylamide based photopolymers have been developed in $1 \mathrm{~mm}$ thick layers by our research team, and these are ideal for use as recording medium in studies relating to hologram recording process $[3,4]$.

The photopolymer used in this study is composed of acrylamide as polymerizable monomer, triethanolamine as radical generator, yellowish eosin as sensitizer and a binder of PVA ${ }^{3}$. We analyze the holographic behavior of the material during recording of diffraction gratings using a continuous argon laser $(514 \mathrm{~nm})$ at an intensity of $5 \mathrm{~mW} / \mathrm{cm}^{2}$ as recording laser. The response of the material is monitored with an He-Ne laser. We study the recording process of diffraction gratings of 1125 lines/mm, in 700-900 $\mu \mathrm{m}$ photopolymer thick layers with PVA Mw $=130000 \mathrm{u}$ binder.

The acrylamide photopolymers with poly(vinyl alcohol) binder are a very versatile materials in the preparation of solid layers with different thickness [5]. Starting in a relatively low thickness, the diffraction gratings registered in these materials show a behavior as volume gratings, still more considering high thickness about $1 \mathrm{~mm}$. In these cases it is easely observed that the diffraction efficiency develops according to Kogelnik expression during recording stage. In the ideal situation, the curve of diffraction efficiency versus energetic exposure has a lineal zone, although it is usual to find examples of deviations to this behavior [6].

Holography: Advances and Modern Trends, edited by Miroslav Miler, Miroslav Hrabovský,

Proc. of SPIE Vol. 7358, 735815 - (c) 2009 SPIE · CCC code: 0277-786X/09/\$18 · doi: 10.1117/12.820449

Proc. of SPIE Vol. 7358 735815-1 
In this investigation we study different cases in which there are deviations to ideal behavior, proposing different hypothesis in order to explain the situations that can be experimentally obtained. A main hypothesis related to Kogelnik theory is presented and the experimental results obtained are according to the theory.

\section{Preparation of the material}

In recording materials based on PVA/AA, a solution of PVA in water forms the matrix and this is used to prepare the solution of monomer AA and the photopolymerization initiator system: yellowish eosin (YE), triethanolamine (TEA). The PVA was supplied by Fluka, AA and TEA by Sigma and YE by Panreac.

We prepare the solutions using a conventional magnetic stirrer, under red light and in laboratory conditions $\left(\mathrm{T}=20{ }^{\circ} \mathrm{C}\right.$, relative humidity $\left.=45 \%\right)$. In Table 1 it can be seen the concentrations of the components in the optimized photopolymer solution which is deposited in order to obtain solid layers of recording media ${ }^{3}$. The solutions are deposited by gravity, in polystyrene circular molds to avoid strain and distortion in the material during the water evaporation process, and left in the dark to allow the water to evaporate, while recording the laboratory conditions ( $T$, relative humidity) during the process. When part of the water has evaporated, the "dry" material is removed from the mold, cut into squares and adhered, without adhesive, to the surface of glass plates measuring $6.5 \times 6.5 \mathrm{~cm}^{2}$. The plates are then ready for exposure, which takes place immediately. The thickness of the material is measured using a conventional micrometer.

Table 1. concentrations of the components of optimized photopolymer solution

\begin{tabular}{lll}
\multicolumn{1}{c}{ Component } & \multicolumn{2}{c}{ Concentration } \\
Poly(vinyl alcohol) & $13.30 \%$ & $\mathrm{w} / \mathrm{v}$ \\
$\mathrm{M}_{\mathrm{w}}=130000 \mathrm{u}$ & & \\
Triethanolamine & 0.15 & $\mathrm{M}$ \\
Acrylamide & 0.34 & $\mathrm{M}$ \\
Yellowish eosin & $9.00 \times 10^{-5}$ & $\mathrm{M}$
\end{tabular}

\section{Holographic set-up}

To study the behavior of the photopolymer as a holographic recording material, we obtained unslanted diffraction gratings using a holographic set-up. The experimental device is shown in Figure 1. An Argon laser at a wavelength of $514 \mathrm{~nm}$ was used to store diffraction gratings by means of continuous laser exposure. The laser beam was split into two secondary beams with an intensity ratio of $1: 1$. The diameters of these beams were increased to $1.5 \mathrm{~cm}$ with an expander, while spatial filtering was ensured. The object and reference beams were recombined at the sample at an angle $\theta\left(16.8^{\circ}\right.$ to the normal $)$ with an appropriate set of mirrors, and the spatial frequency obtained was 1125 lines $/ \mathrm{mm}$. The working intensity at $514 \mathrm{~nm}$ was $5 \mathrm{~mW} / \mathrm{cm}^{2}$. The diffracted and transmitted intensity were monitored in real time with a HeNe laser positioned at Bragg's angle $\left(\theta^{\prime}=20.8^{\circ}\right)$ tuned to $633 \mathrm{~nm}$, where the material is not sensitive. The diffraction efficiency $(\eta)$ was calculated as the ratio of the diffracted beam to the incident power. 


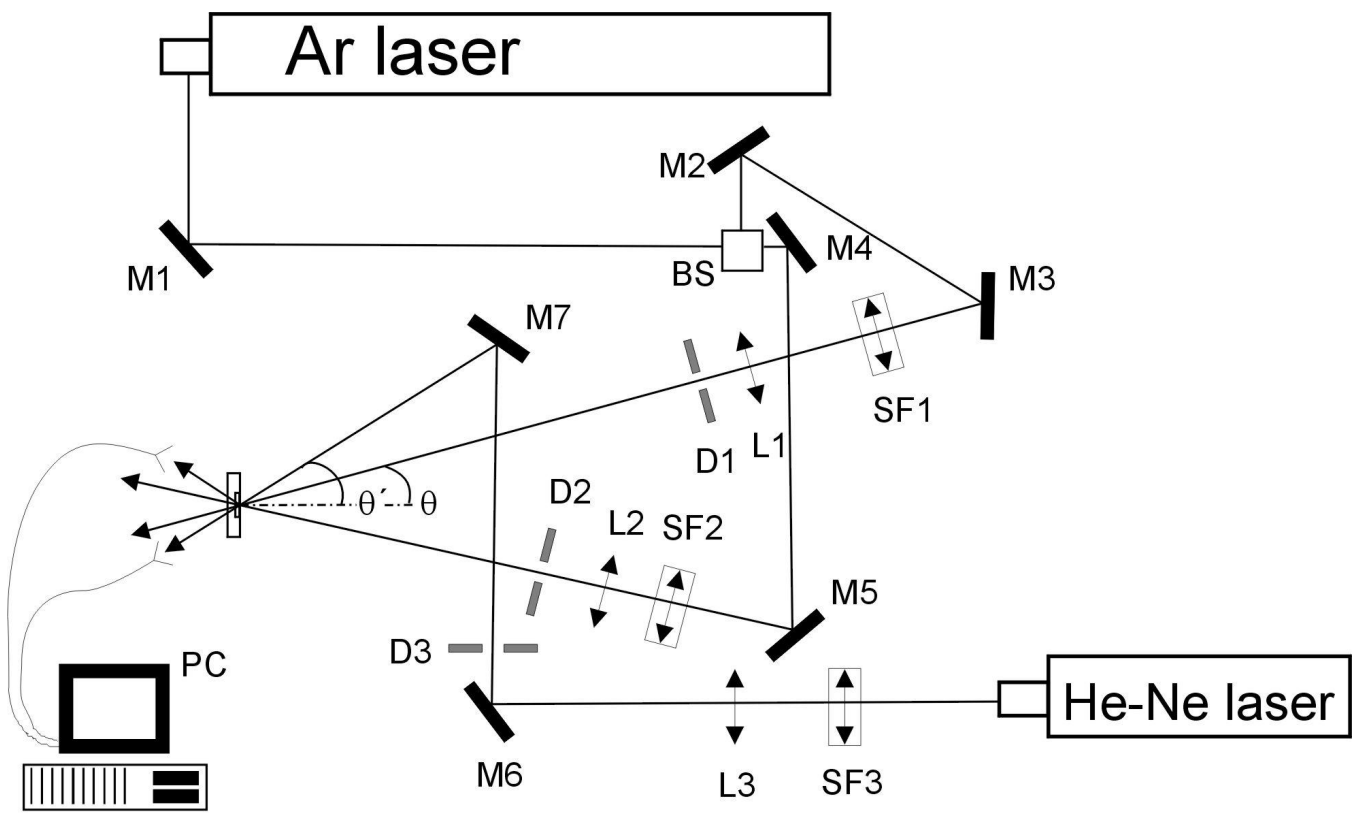

Figure 1. Experimental set-up: BS, beamsplitter, Mi, mirror, SFi, spatial filter, Li, lens, Di, diaphragm, PC, data recorder

\section{Planning the problem}

The experimental data obtained for a $750 \mu \mathrm{m}$ photopolymer layer with a estimated theoretical adjust by Kogelnik, equation 1, are shown in Figure 2 [7].

$$
\eta(t)=\frac{I_{D}}{I_{0}}=\Gamma \operatorname{sen}^{2} \frac{\pi n_{1}(t) d}{\lambda^{\prime} \cos \theta_{i}{ }^{\prime}}
$$

In this equation $\Gamma$ is the absorption, diffusion and reflection losses factor. $\theta_{i}{ }^{\prime}$ is the reconstruction beam angle (Figure 1), measured into the material, which is calculated with refraction Snell law. $\lambda$ ' is the reconstruction beam wavelength. $\mathrm{n}_{1}(\mathrm{t})$ is the refraction index modulation and $\mathrm{d}$ is the diffraction grating thickness [8].

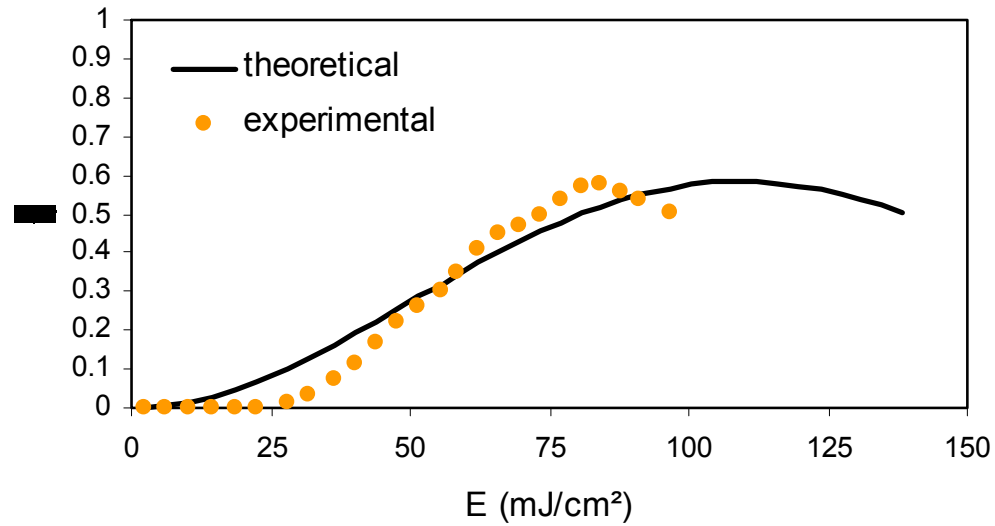

Figure 2. Diffraction efficiency versus exposure for a $750 \mu \mathrm{m}$ photopolymer layer 
It is shown that the behavior of the photopolymer layer is very close to theory, specially in reference to the shape of the diffraction efficiency development during recording except by some details as induction period or inhibition at start of record, factors that are not included in the theoretical expression $^{6}$. In the theoretical adjust the coefficient $\Gamma$ is constant during the recording and the $n_{1}(t) d$ product is proportional to energetic exposure.

In layers with high thickness, it is easy to see an index refraction overmodulation due to the quick raising of $\mathrm{n}_{1}(\mathrm{t}) \mathrm{d}$ product $[9,10]$. Figure 3 is an example of refraction index overmodulation in a $1000 \mu \mathrm{m}$ photopolymer layer and the theoretical adjustment by equation 1 considering the inhibition effects at the start of recording and a linear decreasing of the $\Gamma$ coefficient that is the same to an increasing losses by light diffusion proportional to the index refraction modulation.

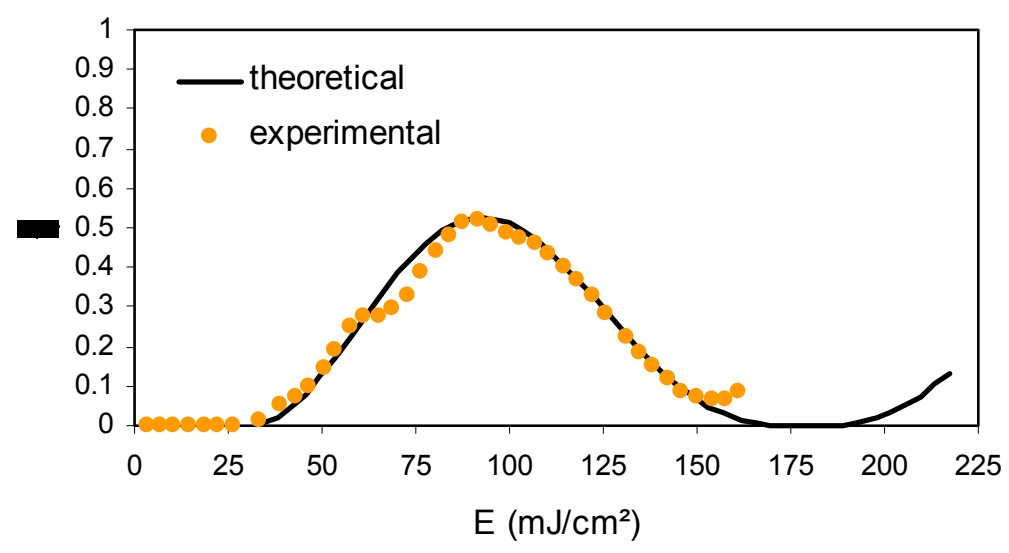

Figure 3. Diffraction efficiency versus exposure for a $1000 \mu \mathrm{m}$ photopolymer layer with refraction index overmodulation

In the ideal situation, the diffraction efficiency versus energetic exposure curve shows a linear zone as it can be seen in Figure 4, which belong to record stage of a diffraction grating in a $700 \mu \mathrm{m}$ photopolymer layer.

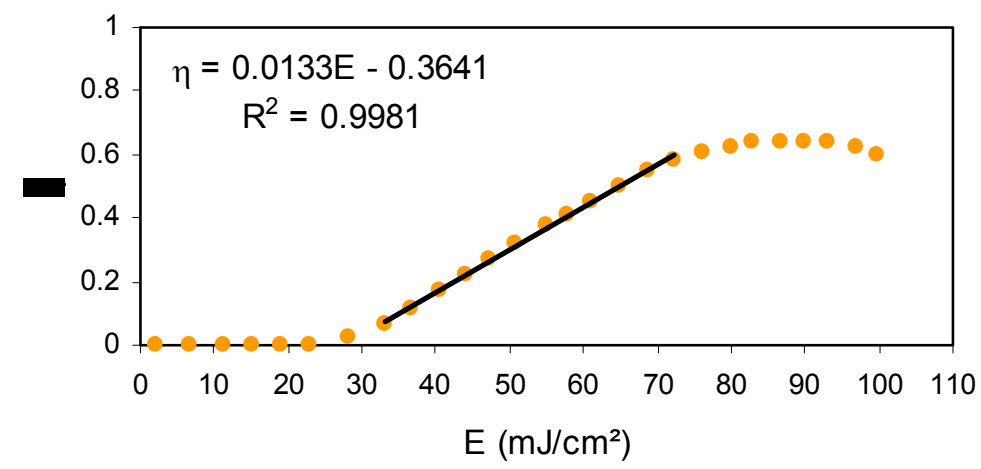

Figure 4. Diffraction efficiency evolution during recording for an ideal situation 
Before the diffraction efficiency reaches a saturation value, there is a zone where diffraction efficiency and energetic exposure is linear, as fit of experimental values shows. This behavior is desirable in most cases, because implies a proportional evolution of diffraction efficiency with energy supplied to the photopolymer, and this is very useful in the practical applications for these materials, for example in recording of holographic optical elements [11]. This type of behavior has occasionally some deviations, therefore in some cases it is not possible to obtain the linear relation that is apointed, as it can be seen for A, B and C curves in Figure 5 for a $900 \mu \mathrm{m}$ photopolymer layer.

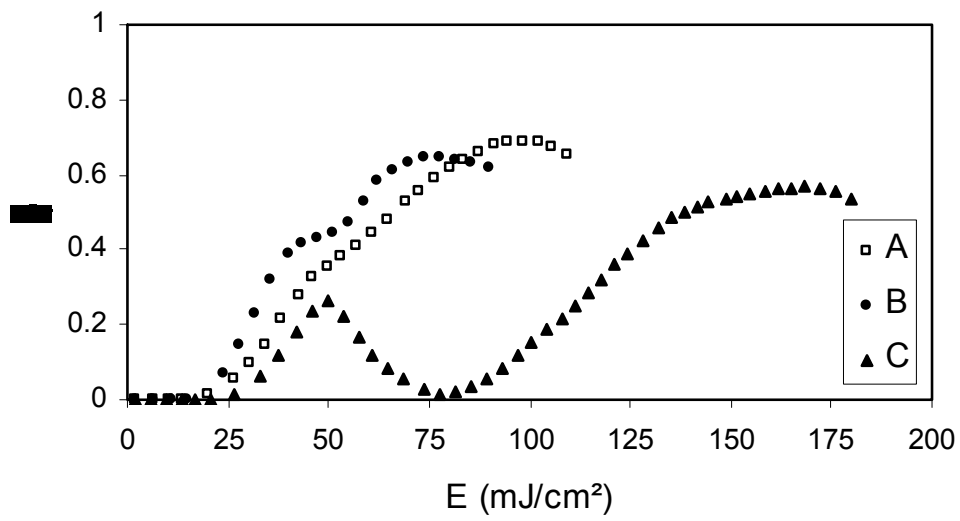

Figure 5. A, Decrease of the slope with an inflection point in the curve. B, decrease of the slope with a transition zone. $\mathrm{C}$, graph with a maximum lower than $\eta \max$

"A" curve correspond to an example of slope change during recording, in this case, for $50 \mathrm{~mJ} / \mathrm{cm}^{2}$ exposure the slope decreases up to saturation. This behavior could imply a polymerization rate decreasing and could be explained by photopolymer properties change during recording, for example, with an increase of the media viscosity, which produces a slow down of the molecular movements $[12,13]$.

A more extreme situation is for " $\mathrm{B}$ " curve, in which exists a transition zone, that it could be a "plateau", where $\eta$ does not increase when the exposure increases. This case has no sense with previous explication, since subsequently the slope is recovered, so in case of photopolymer properties change, due to polymerization, the slope couldn't increase again because the media viscosity increase is proportional to the polymerization progress.

The "C" curve is another different situation that it must not confused with a refraction index overmodulation as in Figure 3. In this case, the curve has a zone in which $\eta$ decreases (in this example down to $\eta \approx 0$ ) before an later increasing up to saturation.

These three examples could be the same phenomenon. Thus, this behavior could be explained by several hypothesis:

Hypothesis 1. The index modulation contribution due to the polymer chains diffusion towards non exposed zones, it will produce the $\eta$ decrease observed, although this it is not possible because the diffusion coefficients of the implied species are not high enough, in this photopolymer formulation, to allow the quick migration to non exposed zones, moreover the $\eta_{\max }$ after recording could quickly decrease down to zero and this it has not seen [14].

Hypothesis 2. Another explanation is also based in diffusion phenomena and considers the influence of monomer concentration modulation in the refraction index modulation. The monomer diffuses from non- 
exposed zones to exposed zones, in which the polymerization takes place, this could imply an additional increase of the refraction index modulation [15].

\section{Results}

We have revised about 300 holograms registered in $1 \mathrm{~mm}$ layers PVA/AA photopolymers made at our laboratory in the last years in order to check the experimental conditions related to these results. The result of $\mathrm{C}$ curve in Figure 5 is obtained mainly when the dye concentration is lower than the optimum and when the layers are not completely dry. This last situation is equivalent to an low dye concentration due to the water of the layer has not completely been evaporated and therefore the dye concentration is lower than expected [3].

When the monomer concentration is very high is also obtained this type of deviations with similar graphs and another more complex with more relative maximums. This have been observed by C. García et al. in PVA/AA photopolymer layers with $150 \mu \mathrm{m}$ thickness [16].

We make 3 photopolymerization experiments without register diffraction gratings, simplifying the experimental set-up in order to check the diffusion hypothesis and the relation with the dye concentration.

\section{Experiment 1}

In this experiment the experimental set-up is simplified using only one recording beam to avoid a possible effect of diffusion in zones with different concentration. A layer without glass support is placed perpendicular to the recording beam. Now there is not a fringe structure in the photopolymer, the monomer and polymer concentrations are homogeneous in the exposed zone and therefore the diffusion effects are not possible. The Figure 6 shows that the transmitted intensity $\mathrm{I}_{\mathrm{T}}$ is increased with the energetic exposure due to the decreasing of the dye concentration.

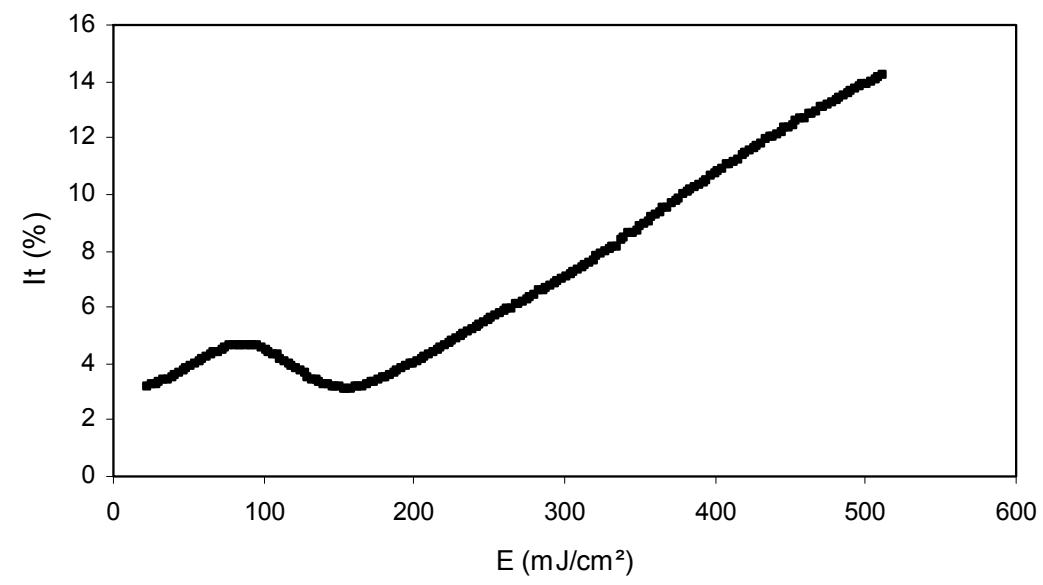

Figure 6. one beam recording in a photopolymer layer without glass plate

At the start of the recording there is a deviation of the linear response that produces a relative maximum in the graph. As in this experiment the diffusion effects are not possible the diffusion hypothesis can be ruled out. 
The exposure to the green light produces a decreasing in the dye concentration which implies a progressive decreasing in the photopolymer absorption and a higher transmission. At the same time the polymerization of the monomer increases the light diffusion that produces a decreasing in the transmission. The last effect is less important than the increase of transmission by the decrease in dye concentration except at the start of recording where the combination of both effects produces the relative maximum. It is important to see that the deviation of the linear response occurs at the start of recording as in the case $\mathrm{C}$ in Figure 5 that we want to explain.

\section{Experiment 2}

In the standard experimental set-up we use now a $633 \mathrm{~nm}$ reconstruction beam and one $514 \mathrm{~nm}$ beam. A 1 $\mathrm{mm}$ thick photopolymer layer is used without glass plate to simplify the system. In Figure 7, the $514 \mathrm{~nm}$ curve shows the transmission intensity percentage by $514 \mathrm{~nm}$ beam multiplied by five to compare both curves. This curve produces the same effect showed in experiment 1 . The $633 \mathrm{~nm}$ curve shows the transmission intensity percentage by $633 \mathrm{~nm}$ beam.

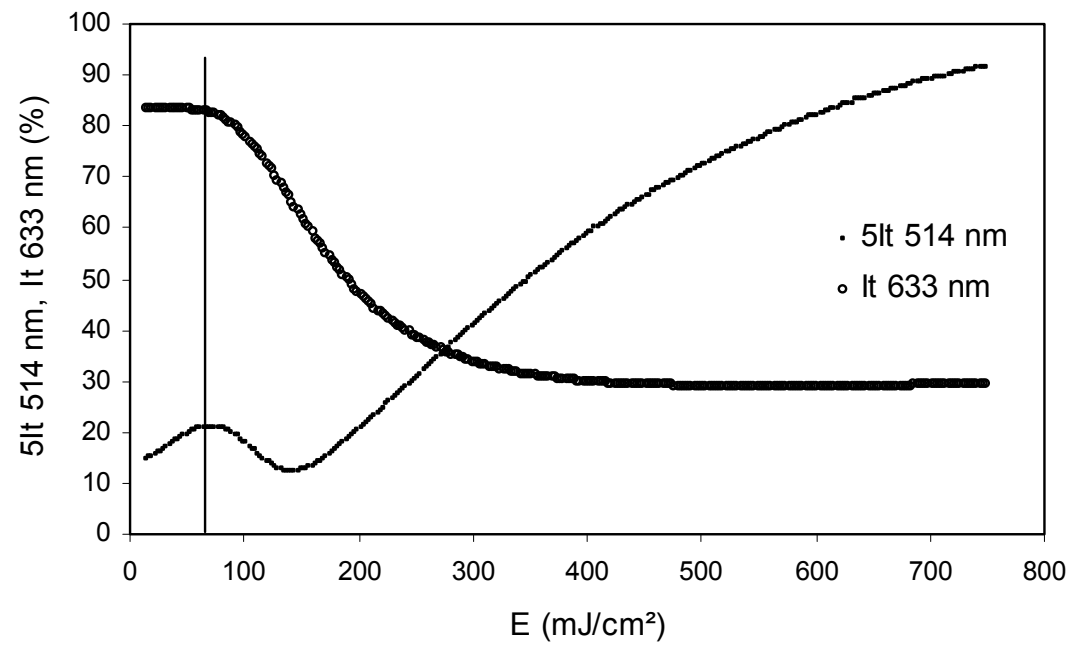

Figure 7. one beam recording in a photopolymer layer without glass plate

In the $633 \mathrm{~nm}$ curve, at the start of recording the transmission is constant because the dye does not absorb light at that wavelength. For the $514 \mathrm{~nm}$ curve, at the start the transmission increases because the dye concentration is reduced by the photopolymerization. When $\mathrm{E}=65 \mathrm{~mJ} / \mathrm{cm}^{2}$, the transmission intensity is maximum $5 \mathrm{I}_{\mathrm{T}}=21 \%$, the dye concentration is decreasing more but now the transmission at $633 \mathrm{~nm}$ decreases by the light diffusion produced by the polymer chains. It can be seen that the start of decreasing of $\mathrm{I}_{\mathrm{T}} 633 \mathrm{~nm}$ match up with the maximum in $\mathrm{I}_{\mathrm{T}} 514 \mathrm{~nm}$ which implies a decreasing in $\mathrm{I}_{\mathrm{T}} 514 \mathrm{~nm}$ to a minimum value $\left(5 \mathrm{I}_{\mathrm{T}}=12 \%, \mathrm{E}=145 \mathrm{~mJ} / \mathrm{cm}^{2}\right)$. From this minimum value the predominant effect is the increase in transmission due to the decreasing in the dye concentration.

The $\mathrm{I}_{\mathrm{T}} 514 \mathrm{~nm}$ varies $2 \%$ and reaches $16.4 \%$ at $600 \mathrm{~mJ} / \mathrm{cm}^{2}$. The deviation of the linear response occurs at the start of recording.

\section{Experiment 3}

We repeat the same experiment 2 but with a photopolymer layer with $1 / 3$ in dye concentration, Figure 8 . Now the $514 \mathrm{~nm}$ curve shows a higher amplitude in the transmission intensity variation and a minor 
transmission at the end of recording. The $\mathrm{I}_{\mathrm{T}} 514 \mathrm{~nm}$ varies $9 \%$ at the relative maximum and reaches $6.4 \%$ at $600 \mathrm{~mJ} / \mathrm{cm}^{2}$. This result is due to a minor dye concentration and therefore the dye concentration can not decrease as in the experiment 2 when the energetic exposure increases. The dye concentration reaches a minimum value at the start of recording and produces a more pronounced effect in the light transmission.

By the other hand, the light diffusion $\left(\Delta \mathrm{R}_{\theta}\right.$ equation 2$)$ is higher than in the experiment 2 because now the dye concentration in low and the polymer molecular weight $\left(\mathrm{M}_{\mathrm{w}}\right)$ is high due to the decreasing in the chain starting moieties number caused by the low dye concentration ${ }^{12}$.

The light diffused by a polymer solution with a molecular weight $\mathrm{M}_{\mathrm{w}}$ can be expressed by equation 2 , in which: polymer concentration (c), light diffusion angle $(\theta)$, solution refraction index (n), incident light wavelength $\left(\lambda_{0}\right)$, polymer chains mean quadratic radius $\left(\mathrm{R}_{\mathrm{G}}{ }^{2}\right)$, system optics characteristics constant $(\mathrm{K})$ and $\mathrm{A} 2, \mathrm{~A} 3, \ldots$ are the virial coefficients $[17,18]$.

The polymer rayleigh relation $\left(\Delta \mathrm{R}_{\theta}\right)$ is proportional to the diffused light at $\theta$ angle divided by incident light. Polymer chains with high $\mathrm{M}_{\mathrm{w}}$ have a high $\Delta \mathrm{R}_{\theta}$ and thus the light diffusion is also high.

$$
\frac{\mathrm{Kc}\left(1+\cos ^{2} \theta\right)}{\Delta \mathrm{R}_{\theta}}=\frac{1}{\mathrm{M}_{\mathrm{w}}}+\frac{16 \pi^{2} \mathrm{n}^{2}}{3 \lambda_{0}^{2} \mathrm{M}_{\mathrm{w}}} \mathrm{R}_{\mathrm{G}}^{2} \operatorname{Sen}^{2} \frac{\theta}{2}+2 \mathrm{~A}_{2} \mathrm{c}+3 \mathrm{~A}_{3} \mathrm{c}^{2}+\ldots
$$

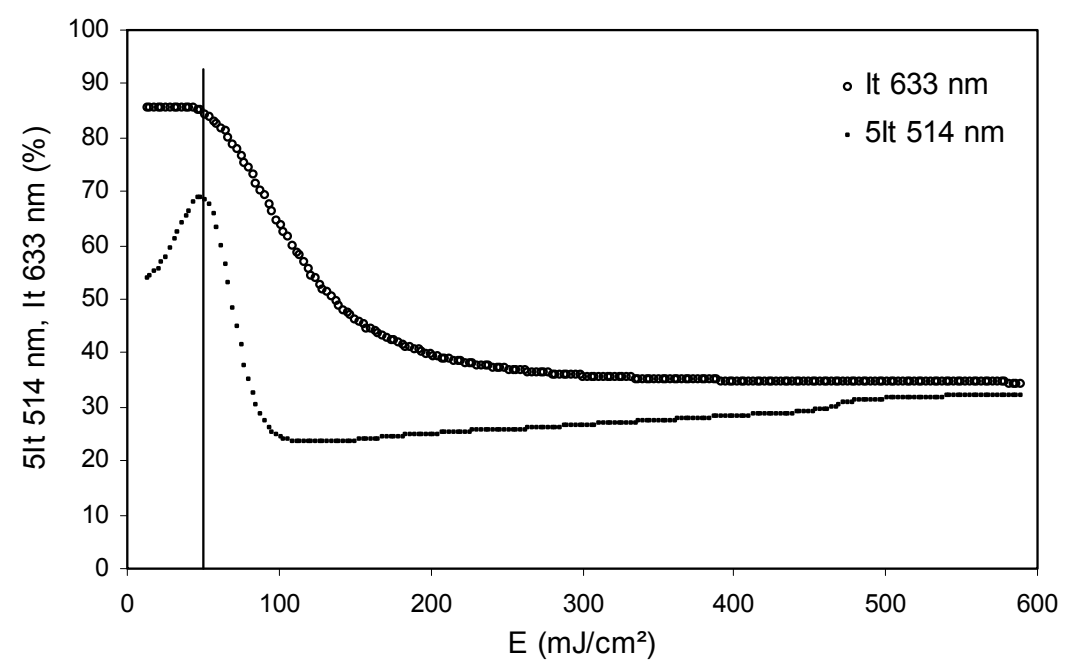

Figure 8 . one beam recording in a photopolymer layer without glass plate, 1/3 dye standard concentration

With the limitation in the dye concentration, the predominant effect is the light diffusion by the polymer chains and therefore the $\mathrm{I}_{\mathrm{T}} 514 \mathrm{~nm}$ is lower than with a normal dye concentration $\left(6.4 \%\right.$ at $600 \mathrm{~mJ} / \mathrm{cm}^{2}$ experiment 3 versus $16.4 \%$ experiment 2).

From experiments made it is deduced that the particular deviations to the photopolymer linear response are produced without polymer diffusion effects, and are related with the dye concentration. A low dye concentration implies a high grating thickness because the light reaches deeper zones in the photopolymer layer due to the high light transmission [8]. By the other hand, a low dye concentration also implies a lower starting polymer chain moieties number y therefore polymer chains with higher molecular weight ${ }^{12}$. Thus, the photopolymer reaches a higher index refraction modulation and grating thickness with a lower 
exposure, at the start of recording. In this situation, the factor $\mathrm{n}_{1}(\mathrm{t}) \mathrm{d}$ from equation 1 must be very high at the start of recording and after increases slowly. We introduces these considerations in equation 1 in order to check if the theoretical expression offers a result according to experimental results in the low dye concentration cases.

In order to simulate theoretically a low dye concentration photopolymer recording we consider the $\mathrm{n}_{1}(\mathrm{t}) \mathrm{d}$ product very high at the start of recording and after an increasing evolution proportional to exposure as shows the indexmodul curve in Figure 5. The indexmodul curve is $5 \times 10^{5}\left(\mathrm{n}_{1}(\mathrm{t}) \mathrm{d}\right)$ to compare the graphs. We also consider light looses increasing depending on exposure, thus the $\Gamma$ factor decreases proportional to exposure [19]. DEsimulation is the diffraction efficiency calculated with equation 1.

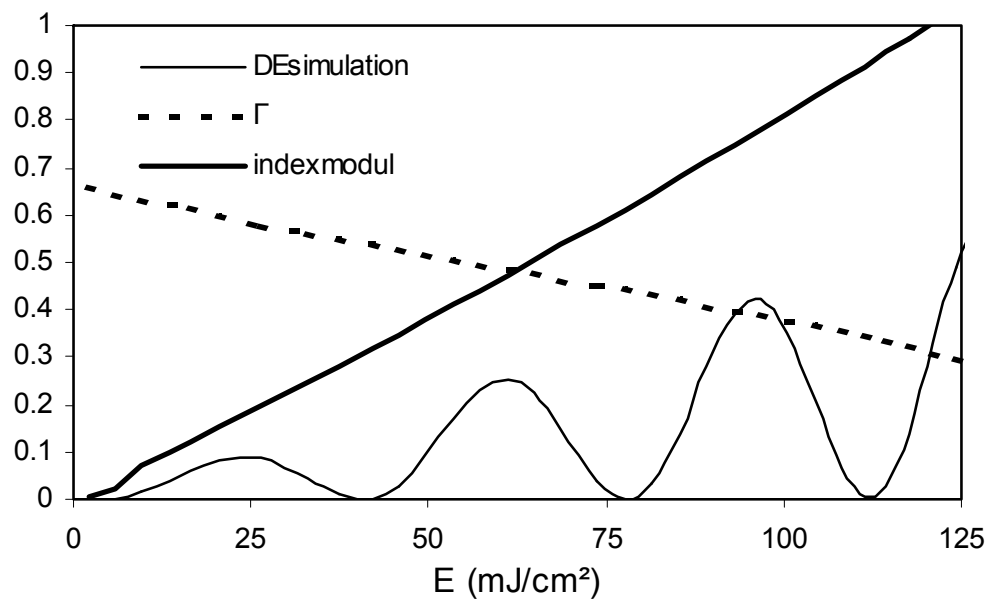

Figure 9. Low dye simulation by equation 1. DEsimulation= diffraction efficiency obtained with equation $1, \Gamma$ decreases proportional to refraction index modulation, indexmodul $=5 \times 10^{5}(\mathrm{n} 1(\mathrm{t}) \mathrm{d}), \mathrm{n}_{1}(\mathrm{t}) \mathrm{d}$ has a light deviation at the start of recording from linear increasing proportional to $\mathrm{E}$.

In DEsimulation it can be seen as the first maximum is lower than the second one and so on. The more important of this simulation is that the relative maximums are increasing progressively with the exposure, which is according to the experimental results with a low dye concentration in which the second maximum is higher than de first one (C curve in Figure 5). It is important emphasize that this theoretical result is not the main result in a hologram recording evolution (Figure 2) and not even an overmodulation (Figure 3).

\section{Conclusion}

Different cases in which there are deviations to ideal behavior during recording of diffraction gratings in photopolymers have been analyzed. The more important deviation experimentally observed and not explained to date is when the first maximum is lower than the second one in the diffraction efficiency versus exposure curve during recording. This situation occurs when the dye concentration in the photopolymer is lower than the optimum value.

The experiments made simplifying the experimental set-up show that there are not diffusion effects implied. By the other hand these experiments prove that the linear deviations are related to the dye concentration. The dye concentration in the photopolymer layer is related to the polymer chains molecular weight and therefore to light diffusion, but also with the grating thickness and with the index refraction modulation material capacity. 
When the photopolymer layer has a low dye concentration, at the start of recording a high index refraction modulation and high grating thickness are produced and thus the $\mathrm{n}_{1}(\mathrm{t}) \mathrm{d}$ product is very high. After, the index modulation and grating thickness increase proportionally to exposure. This produces a first peak with lower diffraction efficiency that the second one in the response curve of the photopolymer. The theoretical simulation by Kogelnik equation with these considerations offers a result according to the experimental.

\section{Acknowledgments}

This work was supported by "Ministerio de Ciencia e Innovación" (FIS2008-05856-C02-01, FIS200805856-C02-02) and "Generalitat Valenciana", Spain (GVPRE/2008/274).

\section{References}

[1] Lessard, R. A. and Manivannan, G., "Holographic Recording Materials: an overview," in Holographic Materials, T. J. Trout, ed., Proc. SPIE 2405, 2-15 (1995).

[2] Martin, S., Leclere, P. E. L. G., Renotte, Y. L. M., Toal, V. and Lion, Y. F., "Characterization of an acrylamide-based dry photopolymer holographic recording material," Opt. Eng. 33, 3942-3946 (1994).

[3] Ortuño, M., Gallego, S., García, C., Neipp, C., Beléndez, A. and Pascual, I., “Optimization of a 1 mm thick PVA/acrylamide recording material to obtain holographic memories: method of preparation and holographic properties," Appl. Phys. B 76, 851-857 (2003).

[4] Ortuño, M., Fernández, E., Gallego, S., Beléndez, A. and Pascual, I., "New photopolymer holographic recording material with sustainable design," Opt. Express 15, 12425-12435 (2007).

[5] Ortuño, M., Gallego, S., García, C., Pascual, I., Neipp, C. and A. Beléndez, "Holographic characteristics of an acrylamide/bisacrylamide photopolymer in 40-1000 $\mu \mathrm{m}$ thick layers," Phys. Scr. T118, 66-68 (2005).

[6] Ortuño, M., Gallego, S., García, C., Neipp, C., and I. Pascual, "Holographic characteristics of a $1 \mathrm{~mm}$ thick photopolymer to be used in holographic memories," Appl. Opt. 42, 7008-7012 (2003).

[7] Kogelnik, H. "Coupled Wave Theory for Thick Hologram Gratings," Bell Sys. Technol. J. 48, 2909 (1969).

[8] Gallego, S., Ortuño, M., Neipp, C., Márquez, A., Beléndez, A., Pascual, I., Kelly, J. and Sheridan, J., "Physical and effective optical thickness of holographic diffraction gratings recorded in photopolymers," Opt. Express, Vol. 13, Issue 6, pp. 1939-1947 (2005).

[9] Gallego, S., Ortuño, M., Neipp, C., García, C., Beléndez, A. and Pascual, I., "overmodulation effects in volume holograms recorded on photopolymers," Opt. Commun. 215, 263-269 (2003).

[10] Neipp, C., Pascual, I. and Beléndez, A., "theoretical and experimental analysis of overmodulation effects in volume holograms recorded on BB-640 emulsions," Journal of optics A: Pure and Appl. Opt. 3, 504-513 (2001).

[11] Márquez, A., Neipp, C., Beléndez, A., Gallego, S., Ortuño, M. and Pascual, I., "edge-enhanced imaging with polyvinyl alcohol/acrylamide photopolymer gratings," Opt. Lett. 28, 1510-1512 (2003).

[12] Odian, G., [Principles of Polymerization], Wiley, New York (1991). 
[13] Kelly, J. V., O’Neill, F. T., Sheridan, J. T., Neipp, C., Gallego, S. and Ortuño, M., "holographic photopolymer materials: nonlocal polymerization driven diffusion under nonideal kinetic conditions," J. Opt. Soc. Am. B 22, 407-416 (2005).

[14] Gallego, S., Ortuño, M., Neipp, C., García, C., Beléndez, A., and Pascual, I., "Temporal Evolution of the Angular Response of a Holographic Diffraction Grating in PVA/Acrylamide Photopolymer," Opt. Express 11, 181-190 (2003).

[15] Gallego, S., Ortuño, M., Neipp, C., Márquez, A., Beléndez, A., Fernández, E., and Pascual, I., “3dimensional characterization of thick grating formation in PVA/AA based photopolymer," Opt. Express 14, 5121-5128 (2006).

[16] García, C., Fimia, A. and Pascual, I., "holographic behavior of a photopolymer at high thicknesses and high monomer concentrations: mechanism of photopolymerization," Appl. Phys. B 72, 311-316 (2001).

[17] Katime, I. and Quintana, J. R., [Scattering properties: light and X-rays in Comprehensive polymer science vol. 1], Pergamon press, Oxford, 103-132 (1989).

[18] Chu., B., [Laser light scattering] Academic Press, New York (1974).

[19] Beléndez, A., Fimia, A., Carretero, L. and Mateos, F., "Self-induced phase gratings due to the inhomogeneous structure of acrylamide photopolymer systems used as holographic recording materials," Appl. Phys. Lett. 67, 3856-3858 (1995). 